\title{
Principais Medicamentos Utilizados no tratamento da Obesidade e Vias de Ação: Uma Revisão Sistemática
}

\author{
Alciêne Maria de Jesus Costa ${ }^{1}$; Stênio Fernando Pimentel Duarte ${ }^{2}$
}

\begin{abstract}
Resumo: A obesidade é considerada um dos mais graves problemas de saúde pública e uma epidemia global, é definida como acúmulo excessivo e anormal de gordura no organismo, que pode atingir níveis capazes de comprometer a saúde em diversas faixas etárias, além disso, poderá influenciar na qualidade de vida de crianças e adultos, estando envolvida em consequências psicológicas e sociais. Diante deste cenário, o presente estudo teve por objetivo fazer uma revisão bibliográfica sobre os principais medicamentos utilizados no tratamento da obesidade e suas vias de ação. A metodologia adotada no presente trabalho foi uma revisão sistemática incluindo artigos sobre os principais fármacos utilizados no tratamento da obesidade. Para esse trabalho foi realizado o levantamento bibliográfico de 40 artigos científicos e foram selecionados 24 artigos dos últimos 6 anos. Foi possível observar nos estudos compilados por esse trabalho, que a maioria dos medicamentos utilizados para o tratamento da obesidade consistem em drogas sintéticas derivadas das anfetaminas que atuam diretamente sobre o sistema nervoso central, mais especificamente sobre as regulações do hipotálamo. O estudo sugere que a atenção farmacêutica é de suma importância para prevenção e promoção da saúde, pois pode proporcionar a redução dos problemas relacionados ao abuso de medicamentos moderadores do apetite,
\end{abstract}

Palavras-chave: Obesidade, Tratamento, femproporex, anfepramona, manzidol, sibutramina e orlistate.

\section{Key Drugs used in the Obesity Treatment and Pathways of Action: A Systematic Review}

\begin{abstract}
Obesity is considered one of the most serious public health problems and a global epidemic is defined as excessive and abnormal accumulation of fat in the body, which can reach levels that can compromise health in various age groups, and may influence the quality Of life of children and adults, being involved in psychological and social consequences. In view of this scenario, the present study aimed to make a bibliographical review on the main drugs used in the treatment of obesity and its pathways of action. The methodology adopted in the present study was a systematic review including articles on the main drugs used in the treatment of obesity. For this work the bibliographical survey of 40 scientific articles was carried out and were selected 24 articles of the last 5 years. It was possible to observe in the studies compiled by this study that the majority of drugs used for the treatment of obesity consist of synthetic drugs derived from amphetamines that act directly on the central nervous system, more specifically on the regulation of the hypothalamus. The study suggests that pharmaceutical care is of paramount importance for prevention and health promotion as it can provide the reduction of problems related to the abuse of appetite moderating drugs,
\end{abstract}

Key words: Obesity, Treatment, femproporex, amfepramone, manzidol, sibutramine and orlistat.

\footnotetext{
${ }^{1}$ Graduação em Farmácia pela Faculdade Independente do Nordeste - FAINOR. Contato: alcienecosta@live.com;

${ }^{2}$ Graduação em Ciências Biológicas na modalidade de Licenciatura Plena pela Universidade do Estado do Rio de Janeiro, mestrado em Ciências (Fisiologia e Fisiopatologia Clínica e Experimental) pela Universidade do Estado do Rio de Janeiro e doutorado em Ciências (Fisiopatologia Clínica e Experimental) pela Universidade do Estado do Rio de Janeiro. Tem experiência na área de Genética, com ênfase em Biologia Molecular e Fisiologia Humana, atuando principalmente com os temas: doenças crônicas, destacando a obesidade humana; polimorfismos genéticos e ataxias espinocerebelares. Atualmente atua como docente da Faculdade Independente do Nordeste - FAINOR e, da Faculdade Tecnologia e Ciências - FTC.
} 


\section{Introdução}

Segundo a Organização Mundial de Saúde (OMS) a obesidade é definida como acúmulo excessivo e anormal de gordura no organismo, que pode atingir níveis capazes de comprometer a saúde em diversas faixas etárias. Além disso, poderá influenciar na qualidade de vida de crianças e adultos, estando envolvida em conseqüências psicológicas e sociais (CASTRO et al., 2016).

A obesidade é considerada um dos mais graves problemas de saúde pública e uma epidemia global (Consenso Latino Americano de Obesidade; CRUZ et al., 2013).Sua prevalência vem crescendo nas últimas décadas e está associada ao desenvolvimento de doenças crônicas não transmissíveis (DCNT) ( MALTA et al., 2014).

Em pesquisa realizada pelo Ministério da Saúde (MS) em 2014, revela que o excesso de peso atinge $52,5 \%$ da população adulta do país. Porém em um levantamento realizado pelo Instituto Brasileiro de Geografia e Estatística (IBGE) em 2015, este índice está próximo de $60 \%$, com prevalência de excesso de peso no sexo feminino $58,2 \%$, e sexo masculino $55,6 \%$ sendo que $24,4 \%$ das mulheres e $16,8 \%$ dos homens apresentam obesidade.

A prevalência de obesidade também está crescendo na infância e adolescência, segundo o IBGE, uma em cada três crianças entre 5 e 9 anos está acima do peso ideal. Em estudos realizados por LEITE et al., 2011, o qual identificou que na faixa etária de 6 a 9 anos foi de $14 \%$ e entre a faixa etária de 10 a 19 anos foi de $19 \%$ sendo que do sexo masculino apresentaram $21 \%$ do percentual e $8 \%$ para o sexo feminino. Em pesquisa, CASTRO et al., 2016, constatouseque dos adolescentes entrevistados, 20\% apresentaram sobrepeso e 30\% obesidade, totalizando excesso de peso em $50 \%$.

A resolução deste problema na infância é fundamental, pois, pode resultar no desenvolvimento de doenças crônicas na fase adulta. A obesidade está relacionada com várias complicações ao indivíduo dentre elas: dificuldades respiratórias, problemas dermatológicos e distúrbios do aparelho locomotor, dislipidemias, doenças cardiovasculares, cirrose, osteoartrite, apnéia do sono (CRUZ et al., 2013; MALTA et al., 2014).

Impedir o crescimento da obesidade em adultos e a sua prevalência em crianças e adolescentes são metas audaciosas estabelecidas no Plano de Ações Estratégicas para o 
enfrentamento das DCNT, na busca por soluções e estratégias para conter o avanço da obesidade, a serem atingidas pelo país até 2022. (MALTA et al., 2014).

De acordo com Cruz e colaboradores (2013) a obesidade é uma doença crônica que quando apenas mudanças no estilo de vida não produzem efeito esperado e/ou quando mais doenças estão relacionadas ou quando houver doenças que predispõem o paciente, faz-se necessário o uso de medicamentos como tratamento complementar, pois a não utilização do fármaco pode agravar o caso colocando em risco a vida do paciente.

Segundo a Agência Nacional de Vigilância Sanitária(ANVISA), o Brasil possui cinco medicamentos registrados e em uso para o tratamento da obesidade:femproporex, anfepramona, manzidol, sibutramina e orlistate. Segue em tabela 1, os medicamentos e as classes os quais pertencem:

Tabela 1: Tratamento medicamentoso da obesidade

\begin{tabular}{ll}
\hline Classe & Medicamento \\
\hline Noradrenérgico & $\begin{array}{l}\text { Femproporex* } \\
\text { Anfepramona* } \\
\text { Manzidol* }\end{array}$ \\
Noradrenérgico e Serotoninérgico & Sibutramina* \\
Inibidor da absorção intestinal de lipídios & Orlistat \\
\hline
\end{tabular}

Fonte: ANVISA, 2014

*Comercialização através da resolução RDC N 50/2014.

Diante deste cenário, o presente estudo teve por objetivo fazer uma revisão bibliográfica sobre os principais medicamentos utilizados no tratamento da obesidade e suas vias de ação.

\section{Metodologia}

A metodologia adotada para a elaboração do artigo foi a de revisão sistemática da literatura, trata-se de um tipo de investigação focada em questão bem definidas, que visa 
identificar, selecionar, avaliar e sintetizar as evidências relevantes disponíveis, ou seja, consiste em um movimento que tem base em critérios pré-determinados e evidências consistentes (SHUTZ; SANT'ANA; SANTOS,2011; GALVÃO; PEREIRA,2014).

Para esse trabalho foi realizado o levantamento bibliográfico de 40 artigos científicos, teses, dissertações, do ano de 2012 a 2017 através da pesquisa nas bases de dados eletrônicas: Literatura Latino-Americana e do Caribe em Ciências da Saúde (LILACS), Scientific Eletronic Library Online (SCIELO), Google Acadêmico e Pubmed. Foram selecionados entre 24 artigos dos últimos 5 anos. Os descritores utilizados na busca foram obesidade, tratamento, medicamentos: femproporex, anfepramona, manzidol, sibutramina e orlistate.

Os critérios de inclusão dos artigos na revisão foram: artigos originais, dos últimos 5 anos de publicação em português, inglês e espanhol.

\section{Resultados e Discussão}

\section{Obesidade}

A obesidade pode ser definida como estado anormal de gordura, que carreta prejuízos à saúde do indivíduo, é também considerada uma doença crônica de difícil controle que, em seu estado mais grave, pode ocorrer falta de sucesso terapêutico além de estar sujeita a recidivas (WINCK et al., 2016; ALMEIDA; ZANATTA; REZENDE,2012).

O parâmetro estipulado pela Organização Mundial de Saúde (OMS) para o diagnóstico da obesidade é o Índice de massa corporal (IMC), obtido a partir da relação entre a estatura ( $\left.\mathrm{m}^{2}\right)$ e o peso corpóreo $(\mathrm{kg})$ do indivíduo em estudo, através deste parâmetro as pessoas cujo IMC encontra-se num valor igual ao maior a $30 \mathrm{~kg} / \mathrm{m}^{2}$ sendo considerado grau 1 da obesidade, grau 2 entre 35 e $39,9 \mathrm{~kg} / \mathrm{m}^{2}$ e grau 3 superior a $40 \mathrm{~kg} / \mathrm{m}^{2}$ (MOREIRA et al.,2012).

Indivíduos cujo o índice de massa corporal (IMC) esteja acima de $40 \mathrm{~kg} / \mathrm{m}^{2}$ são considerados obesos de terceiro grau, pois é forma mais severa da obesidade, nesse grau aumentam os riscos de desenvolver doenças cardiovasculares, alguns tipos de câncer, diabetes, hipertensão arterial, dislipidemias, dificuldade respiratórias, entre outros problemas (MOREIRA et al.,2012;FONSECA-JUNIOR et al.,2013). 
Em relação ao psicológico do indivíduo obeso, a modificação da imagem corporal em consequência do aumento do peso pode ocasionar uma desvalorização do autoconceito e da própria imagem, reduzindo a sua autoestima, e em consequência disso, poderão surgir novos problemas, como depressão, ansiedade, dificultando a relação interpessoal (MARTINS,2012).

Segundo Martins (2012) a obesidade nas últimas décadas tem tomado proporções epidêmicas e a Organização Mundial da Saúde sabe que se não forem tomadas providencias extremas para prevenir e tratar a obesidade, metade da população será obesa em 2025.

Atualmente o tratamento para a obesidade é baseado em medidas não farmacológicas e farmacológicas, as não medicamentosas, incluem terapias comportamentais voltadas para a modificação de hábitos alimentares, atividade física, e orientações de um nutricionista para diminuir o consumo de gordura e calorias, já a medicamentosa é considerada uma terapia adjuvante no tratamento, pois só será utilizado o medicamento caso houver falha terapêutica associada ao tratamento não farmacológico, tenha obesidade graus 2 e 3 ou que tenha alguma outra patologia associada a obesidade (MARTINS et al.,2012).

\section{Tratamento Farmácologico Antiobesidade}

\section{- FEMPROPOREX}

É um medicamento anorexígeno que foi criado como auxiliar no tratamento da obesidade moderada a grave, possui efeitos parecidos com a dextroanfetamina, pois foi sintetizado a partir de modificações na estrutura química da anfetamina visando o efeito anorexígeno e efeito não estimulante, assim, de ínicio, achava-se que o fármaco estaria livre dos efeitos estimulantes das anfetaminas (DA SILVA, 2012; GONÇALVES, 2013; CHAVES, 2016).

O femproporex causa redução do apetite e agudeza pelo odor e sabor, ocasionando uma redução da ingestão de alimentos, pois aumenta a liberação de neurotransmissores e inibe a recaptação de dopamina nos centros hipotalâmicos que regulam o apetite (DA SILVA, 2012; OLIVEIRA DA SILVA;MENDES DA SILVA;OYAMA, 2013; GONÇALVES, 2013; CHAVES, 2016). 
O fármaco em estudo promove alterações neurológicas, comportamentais e cardiovasculares, as reações adversas mais relatadas são hipertensão arterial, pulmonar, náuseas, vômito, ansiedade, vertigem, taquicardia, insônia, excitação, xerostomia (boca seca), alteração da libido, calafrios, palidez, vertigem (PEREIRA et al.,2012).

\section{- ANFEPRAMONA}

A anfepramona (dietilpropiona) foi desenvolvida e lançada no mercado com o intuito de ser um supressor de apetite, sem estimular o sistema nervoso central, entretanto, esse fármaco apresenta estrutura química parecida com a bupropiona e com a estrutura básica dos medicamentos para tratamento antiobesidade com ação central, devido a sua estrutura dietilamina com o anel fenil (DA SILVA,2012; NACCARATO;LAGO,2014; CHAVES,2016).

Este medicamento tem efeitos que parecem ser mediados pela ação dos neurônios dopaminérgicos na fenda sináptica inibindo a recaptação de noradrenalina e aumentando a liberação de dopamina nos receptores pós-sinápticos (DA SILVA,2012; NACCARATO;LAGO,2014; CHAVES,2016)

De acordo com Pereira e colaboradores (2012) a anfepramona pode promover reações adversas como arritmia cardíaca, nervosismo, insônia, alucinações, quadros psicóticos, taquicardia, dor abdominal, vômito, náuseas, diminuição da libido, hipertensão arterial, diarreia, calafrios, palidez, excitação, boca seca, diminuição da potência sexual.

\section{- MANZIDOL}

O manzidol possui estrutura molecular diferente dos medicamentos femproporex e anfepramona que possui estrutura similar a anfetamina, ele é um imidazoisoindol, supressor do apetite trcíclico e tem ação parecida com dois antidepressivos: maprotilina e desipramina (PEREIRA et al.,2012).

O presente fármaco atua bloqueando a recaptação da serotonina e norepirefrina, além de inibir a dopamina, o que ocasiona uma inibição da secreção gástrica o que favorece a supressão do apetite, acredita-se que devido a sua ação no centro da fome, o mazindol promove 
uma supressão do apetite pela diminuição da ingestão alimentar, inibição da absorção da glicose e aumento da atividade locomotora (DUTRA, SOUZA, PEIXOTO, 2015; CHAVES, 2016).

As reações adversas mais comum do mazindol são insônia, boca seca, nervosismo, cefaléia, arrepios, náuseas, palpitações, fraqueza, vertigem, constipação, fraqueza

\section{- SIBUTRAMINA}

A sibutramina é o primeiro fármaco utilizado no tratamento antiobesidade, inicialmente foi desenvolvida como antidepressivo, entretanto, durantes alguns testes foi verificado que este era capaz de reduzir o apetite (DEFÁVERI, 2012; DE BRITO;RABELO,2013). Esse medicamento é uma monoamina que atua inibindo a recaptação de três neurotransmissores, são eles: serotonina, noradrenalina e a dopamina, fazendo com que essas substâncias fiquem livres por mais tempo excitando os neurônios (LONDOÑO-LEMOS, 2012; DEFÁVERI et al., 2012; DE BRITO;RABELO, 2013).

De acordo com Londoño-Lemos (2012) e Franco (2012) a sibutramina vem demonstrando vários efeitos colaterais, sendo o mais comum a sensação de boca seca, embora outros efeitos como insônia, pressão arterial alta e aumento da frequencia cardíaca,diarréia, artrite, dor de cabeça, constipação, anorexia e naúseas também sejam frequentes.

\section{- ORLISTATE}

Orlistate é um potente inibidor exclusivo de lipases gástricas e pancreáticas, atua especificamente no lúmen intestinal formando uma ligação covalente com o sítio serina ativo das lipases gastrointestinais, assim a enzima inativada impede a hidrolise de gordura dietética em 30\%, logo diminui a absorção de gordura eliminando-a nas fezes (OLIVEIRA DA SILVA, 2013; LUNAGARIYA et al., 2014; RIBEIRO, 2014).

Os principais efeitos colaterais do orlistate incluem diarréia, diminuição da absorção de vitaminas lipossolúveis, dores abdominais, flatulência, incontinência (RIBEIRO, 2014).

A tabela 1 exibe de forma sintetizada a abordagem dos autores que relatam sobre os medicamentos antiobesidade mais utilizados: 
Tabela 1 - Revisão da literaturamedicamentos antiobesidade mais prescritos

Autores

(CARVALHO E MARTINS et al.,2011)

(ZUBARANI;LAZZARETI,2013)

\section{Principais achados em seus estudos}

Avaliou o uso de fármacos antiobesidade entre estudantes, observou-se que as anfetaminas e as aminas simpaticomiméticas $(40,5 \%)$ foram as drogas mais usadas e entre aqueles que referiram uso de agentes antiobesidade, $62,2 \%$ eram do sexo feminino. $\mathrm{O}$ uso desses medicamentos se mostrou preocupante, principalmente pela elevada proporção de uso sem indicação ou prescrição médica.

Estabeleceu a prevalência do uso de moderadores de apetite entre estudantes da área da saúde, pode-se concluir que uma significativa porcentagem da amostra $(15 \% ; n=45)$ usou moderadores de apetite pelo menos uma vez na vida e as substâncias mais comumente usadas foram drogas estimulantes (5\%), incluindo amfepramona $(3,3 \%)$ e femproporex $(1,7 \%)$. Pode-se concluir que as drogas estimulantes simpatomiméticas foram os agentes mais comumente usados, principalmente entre os alunos de nutrição e enfermagem.

Avaliou os principais inibidores de apetite prescritos, e conclui-se que o Femproporex (43\%) foi o fármaco mais prescrito durante o período analisado, sendo os maiores consumidores as mulheres (82\%). O estudo detectou a alta utilização desta classe de medicamentos, sendo importante o controle adequado do uso destes anorexígenos conforme preconizado atualmente pelas agências reguladoras.

FONTE: Da autora.

De acordo com os dados obtidos na tabela o femproporex é o fármaco mais prescrito por possuir menor efeito colateral em relação aos demais fármacos, ou seja, é o moderador de apetite mais utilizado e um dos mais bem tolerados (PEREIRA et al.,2012). 


\section{Conclusão}

É possível observar nos estudos compilados por esse trabalho, que a maioria dos medicamentos utilizados para o tratamento da obesidade consistem em drogas sintéticas derivadas das anfetaminas que atuam diretamente sobre o sistema nervoso central, mais especificamente sobre as regulações do hipotálamo.

É importante ressaltar o aumento da prevalência da obesidade do país como fator de suma importância na saúde pública, e também, deve-se atentar para o abuso na automedicação de anorexígenos, como demonstrado no presente trabalho, causa diversos efeitos colaterais.

O estudo sugere que a atenção farmacêutica é de suma importância para prevenção e promoção da saúde, pois pode proporcionar a redução dos problemas relacionados ao abuso de medicamentos moderadores do apetite, cabendo ressaltar os riscos da automedicação não só desses medicamentos, mas de outras substâncias também.

\section{Referências}

CARVALHO E MARTINS,M.C.;SOUZA FILHO,M.D.;DE CARVALHO,J.S.R.; MULLER,M.C.; NEVES,R.V.;MOUSINHO,P.C.;LIMA,I.P. Uso de drogas antiobesidade entre estudantes universitários. RevAssocMedBras, n.57,n.5,p.570-576,2011.

CASTRO,G.G.; FIGUEIREDO,G.L.A.;DA SILVA, T.S.; DE FARIA, K.C.Qualidade de vida em crianças escolares com sobrepeso e obesidade. Rev. do Departamento de Educação Física e Saúde e do Mestrado em Promoção da Saúde.,v.17, 2016.

CHAVES,A.S.Estabelecimento de um modelo experimental de obesidade em camundongos para estudo da atividade farmacológica de fármacos sintéticos e naturais com ação antiobesidade. Programa de Pós-Graduação em Vigilância Sanitária, Instituto Nacional em Controle de Qualidade em Saúde. Fundação Oswaldo Cruz, 2016, 54 f.: il., tab.

CRUZ,A.C.S.;SANTOS.E.N. Avaliação de medicamentos para emagrecer em farmácias, no município de Ceres- Goiás, Brasil. Rev. Da Universidade Vale do Rio Verde.,v.10,n.1.p 402$409,2013$.

DE BRITO, A.F.;RABELO,M.M.; Avaliação do índice de venda da sibutramina na cidade de Ceres-GO. Revista Eletrônica da Faculdade de Ceres,v.2,n.1,2013. 
DEFÁVERI,M.A.S.; LAPORTA,L.V.; DOS SANTOS,M.R.; DA SILVEIRA, R.L.;ALVES,M.P.; RUBIM,A.M. Avaliação da qualidade das cápsulas de cloridrato de sibutramina manipuladas em farmácias. Disc. Scientia. Série: Ciências da Saúde, Santa Maria, v. 13, n. 1, p. 71-83, 2012.

DUTRA,J.R.;SOUZA,S.M.F.;PEIXOTO,M.C. Influência dos padrões de beleza veiculados pela mídia, como fator decisório na automedicação com moderadores de apetite por mulheres no município de miracema-RJ. Universidade Iguaçu Campus, ,7 $7^{\mathrm{a}}$ ediç̧ão, Rio de Janeiro, 2015.

FONSECA-JUNIOR, S.J.; SÁ, C.G.A.B.; RODRIGUES, P.A.F.; OLIVEIRA, A.J.; FERNANDES-FILHO,J. Physicalexerciseandmorbidobesity: a systematicreview. Arquivo brasileiro de cirurgia digestiva, São Paulo, vol.26,n.1, 2013.

FRANCO,R.R.O efeito da Sibutramina na perda de peso de adolescentes obesos. Dissertação(mestrado)-USP-FM-DBD-313/12, São Paulo,2012.

GALVÃO,T.F.; PEREIRA,M.G.; Revisões sistemáticas da literatura: passos para sua elaboração. Epidemiol. Serv. Saúde, Brasília, v.23, n.1, p.183-184, 2014.

LEITE,F.R.M; NAVARRO,A.C. Prevalência de sobrepeso e obesidade em crianças e adolescentes freqüentadores de um programa sócio-educativo de uma ONG na zona oeste de São Paulo. Rev. Brasileira de Obesidade, Nutrição e Emagrecimento.,v.5,n.26, p. 80-89, 2011.

LONDOÑO-LEMOS,M.E.Tratamiento farmacológico contra laobesidad. Rev. Colomb. Cienc. Quim. Farm., v.4,n. 2, p. 217-261, 2012.

LUNAGARIYA,N.A.;PATEL,N.K.;JAGTAP,S,C.;BHUTANI,K.K.Inhibitorsofpancreatic lipase: stateoftheartandclinical perspectives. EXCLI Journal, v.13,p.897-921,2014.

MALTA,D.C.; DA SILVA JR,J.B.Plano de ações estratégicas para o enfrentamento das doenças crônicas não transmissíveis no Brasil após três anos de implantação, 2011-2013. Epidemiol. Serv. Saúde, 2014.

MARTINS， E..L.M.;DO AMARAL,M.P.H.; FERREIRA,M.B.C.;PEREIRA,M.C.S.; PEREIRA CAMPOS,D.;RIBEIRO,D.C. Dispensações de psicotrópicos anorexígenos no município de Juiz de Fora, Minas Gerais, Brasil. Ciência \& Saúde Coletiva, v.17,n.12,p. 33313342, 2012.

MARTINS,S.O peso da mente feminina: associação entre obesidade e depressão. Rev. Por. Med, Geral Farm, 2012, v.8, p.163-6.

MOREIRA,P.;ROMUALDO,M.C.S;AMPARO,F.C.;PAIVA,C.;ALVES,R.;MAGNONI,D.;K OVACS,C. A educação nutricional em grupo e sua efetividade no tratamento de pacientes 
obesos. Revista Brasileira de Obesidade, Nutrição e Emagrecimento, São Paulo ,v.6, n.35, p.216-224, 2012. ISSN 1981-9919.

NACCARATO,M.C.;LAGO,E.M.O. Uso dos anorexígenos anfepramona e sibutramina: benefício ou prejuízo à saúde? Revista Saúde, Guarulhos, v.8, n. 1/2, 2014.

OLIVEIRA DA SILVA,L.F.; MENDES DA SILVA,F.V.; OYAMA,S.M.R. Prevalência do uso de medicamentos para emagrecer entre universitárias. Revista Recien. São Paulo, v.3,n.7,p.1926,2013 .

PEREIRA,F.A.;NEVES,E.B.;MASCARENHAS,L.P.;PIETROVSKI,E.F.; Avaliação de prescrições de anorexígenos em farmácia magistral. Rev. Bras. Farm. v.93,v.4,p. 481-486, 201.

RIBEIRO,J.R.Avaliação dos efeitos da Bidens pilosa L., BrassicacampestrisL. e sua associação sobre a obesidade induzida por dieta hipercalórica. Dissertação (mestrado) UNESP - Campus Botucaru,Botucatu, 2014.

WINCK, A.D.; HEINZAMANN-FILHO,J.P.; SOARES,R.B.;DA SILVA,J.S.; WOSZEZENKI,C.T.; ZANATTA,L,B. Efeitos da obesidade sobre os volumes e as capacidades pulmonares em crianças e adolescentes: uma revisão sistemática. Rev. Paul Pediatr. 2016,v.34,n.4,p.50-517.

ZUBARANI,C.; LAZZARETI,R.Uso de moderadores de apetite entre estudantes da área da saúde na Região Sul do Brasil. Einstein,v.11,n.1,p:47-52, 2013.

Como citar este artigo (Formato ABNT):

COSTA, Alciêne M.J.; DUARTE, Stênio F.P. Principais Medicamentos Utilizados no tratamento da Obesidade e Vias de Ação: Uma Revisão Sistemática. Id on Line Revista Multidisciplinar e de Psicologia, Maio de 2017, vol.11, n.35, p.199-209. ISSN: 1981-1179.

Recebido: 18.05.2017

Aceito: 24.05 .2017 